\title{
Improved precision on the radius of the nearby super-Earth 55 Cnc e $^{\star}$
}

\author{
M. Gillon ${ }^{1}$, B.-O. Demory ${ }^{2}$, B. Benneke ${ }^{2}$, D. Valencia ${ }^{2}$, D. Deming ${ }^{3}$, S. Seager ${ }^{2}$, Ch. Lovis ${ }^{4}$, \\ M. Mayor ${ }^{4}$, F. Pepe ${ }^{4}$, D. Queloz ${ }^{4}$, D. Ségransan ${ }^{4}$, and S. Udry ${ }^{4}$ \\ ${ }^{1}$ Institut d'Astrophysique et de Géophysique, Université de Liège, Allée du 6 Août 17, Bât. B5C, 4000 Liège, Belgium \\ e-mail: michael.gillon@ulg.ac . be \\ 2 Department of Earth, Atmospheric and Planetary Sciences, Department of Physics, Massachusetts Institute of Technology, \\ 77 Massachusetts Ave., Cambridge, MA 02139, USA \\ 3 Department of Astronomy, University of Maryland, College Park, MD 20742-2421, USA \\ 4 Observatoire de Genève, Université de Genève, 51 chemin des Maillettes, 1290 Sauverny, Switzerland
}

Received 21 October 2011 / Accepted 28 December 2011

\begin{abstract}
We report on new transit photometry for the super-Earth 55 Cnc e obtained with Warm Spitzer/IRAC at $4.5 \mu$ m. An individual analysis of these new data leads to a planet radius of $2.21_{-0.16}^{+0.15} R_{\oplus}$, which agrees well with the values previously derived from the MOST and Spitzer transit discovery data. A global analysis of both Spitzer transit time-series improves the precision on the radius of the planet at $4.5 \mu \mathrm{m}$ to $2.20 \pm 0.12 R_{\oplus}$. We also performed an independent analysis of the MOST data, paying particular attention to the influence of the systematic effects of instrumental origin on the derived parameters and errors by including them in a global model instead of performing a preliminary detrending-filtering processing. We deduce an optical planet radius of $2.04 \pm 0.15 R_{\oplus}$ from this reanalysis of MOST data, which is consistent with the previous MOST result and with our Spitzer infrared radius. Assuming the achromaticity of the transit depth, we performed a global analysis combining Spitzer and MOST data that results in a planet radius of $2.17 \pm 0.10 R_{\oplus}$ $(13820 \pm 620 \mathrm{~km})$. These results point to $55 \mathrm{Cnc}$ e having a gaseous envelope overlying a rocky nucleus, in agreement with previous works. A plausible composition for the envelope is water which would be in super-critical form given the equilibrium temperature of the planet.
\end{abstract}

Key words. techniques: photometric - binaries: eclipsing - planetary systems - stars: individual: 55 Cnc

\section{Introduction}

Transiting planets are of fundamental interest for the field of exoplanetary science. Their advantageous geometrical configuration relative to Earth enables the thorough study of their physical, orbital and atmospheric properties, provided they orbit around stars bright enough to permit high signal-to-noise follow-up observations. For a given stellar type, this last condition is drastically more stringent for terrestrial planets than for gas giants, leading to the conclusion that only the handful of solid planets that should transit stars of the closest solar neighborhood would be suitable for a thorough characterization with existing or future instruments (e.g. Seager et al. 2009).

In this context, two teams, including ours, independently announced the first transit detection for a solid planet orbiting a nearby star visible to the naked eye (Winn et al. 2011, hereafter W11; Demory et al. 2011, hereafter D11). This transiting "superEarth", 55 Cnce, is the inner most of the five planets currently known to orbit around 55 Cancri, a G8-K0 dwarf star located at only 41 light-years from Earth (see D11 and references therein). We detected one of its transits with Spitzer, allowing us to deduce a radius of $2.08_{-0.16}^{+0.17} R_{\oplus}$ and a mass of $7.81_{-0.53}^{+0.58} M_{\oplus}$ for the planet (D11). Together, these values favor a solid planet with a

* The photometric time series used in this work are only available at the CDS via anonymous ftp to

cdsarc.u-strasbg.fr (130.79.128.5) or via

http://cdsarc.u-strasbg.fr/viz-bin/qcat?]/A+A/539/A28 significant fraction of ice. On their side, Winn et al. detected several transits of $55 \mathrm{Cnc}$ e with the MOST satellite and reported a planet radius consistent with ours, $2.00 \pm 0.14 R_{\oplus}$ (W11). Soon after this double transit detection, a third team reported impressively precise values for the host star's parameters based on new interferometric observations (von Braun et al. 2011, see Table 1). Thanks to these last results, made possible by the brightness of the star, our knowledge of the mass and size of $55 \mathrm{Cnc}$ e is only limited by the precision of the radial velocities and transit photometry gathered so far.

Aiming to pursue the characterization of this fascinating planet, we monitored another of its transits with Spitzer in our program dedicated to the search of nearby transiting low-mass planets (ID 60027). In the next two sections, we present these new data and their analysis, including a global analysis of MOST and Spitzer photometric time-series aiming to determine the size of the planet as precisely as possible. We discuss our results and their implications in the last section of the paper.

\section{New Warm Spitzer transit photometry}

We monitored 55 Cnc with Spitzer on 20 June 2011 from 09h08 to $15 \mathrm{~h} 02 \mathrm{UT}$, corresponding to a transit window of $55 \mathrm{Cnce}$ as computed from our transit ephemeris presented in D11. The IRAC detector acquired 6230 sets of 64 subarray images at $4.5 \mu \mathrm{m}$ with an integration time of $0.01 \mathrm{~s}$. These 6230 sets were calibrated by the Spitzer pipeline version S18.18.0 and are 

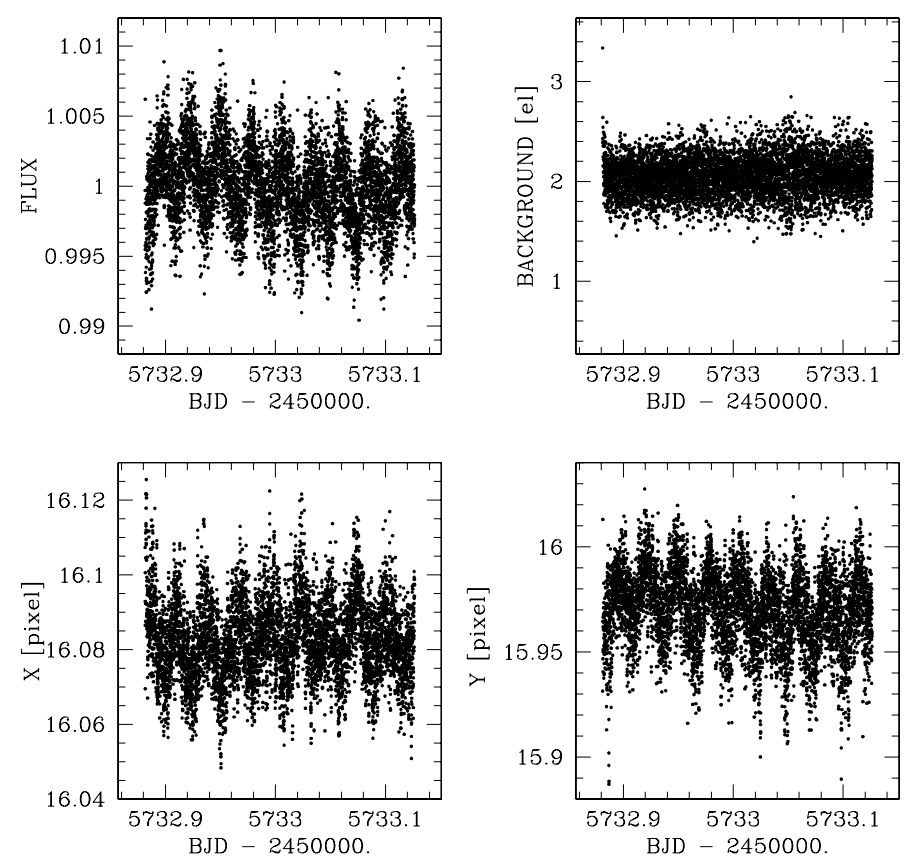

Fig. 1. Top left: raw light curve obtained for $55 \mathrm{Cnc}$ with Warm Spitzer at $4.5 \mu \mathrm{m}$. Top right: corresponding background time-series. Bottom: corresponding time-series for the $x$ (left) and $y$ (right) positions of the stellar center. The correlation between measured stellar counts and the stellar image positions is clearly noticeable. This "pixel-phase" effect is well-known for the InSb Spitzer/IRAC detectors (e.g. Knutson et al. 2008).

available on the Spitzer Heritage Archive database ${ }^{1}$ under the form of basic calibrated data (BCD) files. Our reduction of these data was identical to the one presented in D11 and we refer the reader to this paper for details. Figure 1 shows the resulting raw light curve composed of 6197 flux measurements, and also the time-series for the background counts and the $x$ and $y$ positions of the target's point-spread function (PSF) on the detector array. We can notice from Fig. 1 that the background counts remained stable during the whole run, unlike during our first transit observation (see D11, Fig. 1).

\section{Data analysis}

\subsection{Individual analysis of the new Warm Spitzer data}

In a first step, we performed an individual analysis of our new data. We used for this purpose our adaptative Markov-Chain Monte Carlo (MCMC) code (see D11 and references therein for details). Gaussian priors assumed for the stellar parameters are shown in Table 1. Uniform priors were assumed for the other parameters of the system. First, we performed a thorough model comparison, performing for each tested model a MCMC analysis composed of one chain of 10000 steps and deriving the model marginal likelihood from the MCMC outputs using the method described by Chib \& Jeliazkov (2001). Each model was composed of a baseline representing the low-frequency instrumental and stellar effects multiplied by a transit model computed under the formalism of Mandel \& Agol (2002) assuming a quadratic limb-darkening law. The baseline models included an $x$ - and $y$-position polynomial (D11, Eq. (1)) to model Warm Spitzer "pixel-phase" effect (see Fig. 1), which we added as needed to one or several functions of time. At the end, more than 30 models

\footnotetext{
${ }^{1}$ http://sha.ipac. caltech.edu/applications/Spitzer/SHA
}

Table 1. Gaussian priors assumed in this work for the stellar parameters.

\begin{tabular}{lc}
\hline \hline Mass $\left[M_{\odot}\right]$ & $0.905 \pm 0.015^{1}$ \\
Radius $\left[R_{\odot}\right]$ & $0.943 \pm 0.010^{1}$ \\
$T_{\text {eff }}[\mathrm{K}]$ & $5196 \pm 24^{1}$ \\
Metallicity $[\mathrm{Fe} / \mathrm{H}][$ dex] & $+0.31 \pm 0.04^{2}$ \\
Limb-darkening linear parameter $u_{1,4.5} \mu \mathrm{m}$ & $0.0711 \pm 0.0009^{3}$ \\
Limb-darkening quadratic parameter $u_{2,4.5} \mu \mathrm{m}$ & $0.1478 \pm 0.0020^{3}$ \\
Limb-darkening linear parameter $u_{1, \mathrm{MOST}}$ & $0.657 \pm 0.090^{4}$ \\
Limb-darkening quadratic parameter $u_{2, \mathrm{MOST}}$ & $0.115 \pm 0.045^{4}$ \\
\hline
\end{tabular}

References. ${ }^{(1)}$ von Braun et al. (2011); (2) Valenti \& Fischer (2005); (3) Claret \& Bloemen (2011); ${ }^{(4)}$ W11.

were tested, the one corresponding to the highest marginal likelihood has as baseline a second-order position polynomial added to a fourth-order time polynomial and to a sinusoid. Because the Bayes factor (e.g. Carlin \& Louis 2008) between this model and the second most likely model is about 100 , we selected it for sampling the posterior probability density distributions of the transit parameters. We performed a new MCMC analysis for this purpose composed of two chains of 100000 steps, and checked their convergence using the statistical test of Gelman \& Rubin (1999). Table 2 provides the resulting values and errors for the transit parameters, while Fig. 2 shows the best-fit global model superimposed on the data and the best-fit transit model superimposed on the data divided by the best-fit baseline model.

Comparing the resulting transit parameters shown in Table 2 to those derived in D11, we notice that the derived transit depths agree at better than $1 \sigma\left(463_{-57}^{+64} \mathrm{ppm}\right.$ here vs. $410 \pm 63 \mathrm{ppm}$ for D11) but that the agreement for the derived impact parameters is only of $\sim 2.8 \sigma\left(0.509_{-0.074}^{+0.056}\right.$ here vs. $0.16_{-0.13}^{+0.10}$ for D11). We suspect that this discrepancy comes mostly from the instrumental effect that affected our first transit data. Indeed, in D11 we had to model a sharp increase of the effective gain of the detector during the run that was correlated to a strange behavior of the background counts. We encountered this systematic effect in other Warm Spitzer time-series acquired in our program 60027 (Gillon et al., in prep.). Unfortunately, this effect occurred just during the egress of the transit, and we suspect that it could have biased the derived marginalized posterior distribution function for the transit impact parameter.

The derived period and amplitude for the sinusoid function of the baseline model are $59 \pm 2$ min and $107 \pm 24$ ppm, consistent with the values derived in D11 for the first Spitzer transit, $51 \pm 3 \mathrm{~min}$ and $115 \pm 27 \mathrm{ppm}$. The origin of this low-amplitude periodic effect is still unclear (see D11).

\subsection{Global analysis of Warm Spitzer data}

Aiming to minimize the impact of Warm Spitzer instrumental effects and to obtain the strongest constraints on the planet's infrared radius, we then performed a global MCMC analysis using as input data the two Spitzer light curves. We assumed for both of them the same baseline model as used for their individual analysis, with the exception that we did not fix the start time of the effective gain increase for the first transit, but instead let it be a free parameter to take into account our limited understanding of this systematic effect. The Gaussian prior $P=0.7365437 \pm 0.000052$ days based on the RV data analysis presented in D11 was used for the orbital period of the planet. The MCMC was composed of two chains of 100000 steps. 
Table 2. Median and 1- $\sigma$ limits of the marginalized posterior distributions obtained for the parameters of 55 Cnc e from our MCMC analysis of the Warm Spitzer data.

\begin{tabular}{|c|c|c|c|c|c|}
\hline Parameter & Spitzer transit 2 & Spitzer transit $1 \& 2$ & MOST & Spitzer + MOST & Unit \\
\hline Transit timing $T_{\mathrm{tr}}$ & $5733.0094_{-0.0011}^{+0.0012}$ & $5733.0085_{-0.0014}^{+0.0011}$ & $5607.0584_{-0.0017}^{+0.0016}$ & $5733.0087_{-0.0011}^{+0.0013}$ & $\mathrm{BJD}_{\mathrm{TDB}}-2450000$ \\
\hline Orbital period $P$ & 0.7365437 (fixed) & $0.7365460_{-0.0000046}^{+0.000049}$ & $0.7365437 \pm 0.0000052$ & $0.7365449_{-0.0000050}^{+0.000046}$ & days \\
\hline Transit depth $\left(R_{\mathrm{p}} / R_{*}\right)^{2}$ & $463_{-54}^{+57}$ & $458 \pm 47$ & $394_{-51}^{+61}$ & $447_{-38}^{+40}$ & ppm \\
\hline Planet-to-star radius ratio $\left(R_{\mathrm{p}} / R_{*}\right)$ & $0.0215 \pm 0.0013$ & $0.0214 \pm 0.0011$ & $0.0198_{-0.0013}^{+0.0015}$ & $0.02113_{-0.00091}^{+0.00093}$ & \\
\hline Transit circular impact parameter $b$ & $0.509_{-0.074}^{+0.056}$ & $0.500_{-0.085}^{+0.057}$ & $0.44_{-0.16}^{+0.11}$ & $0.459_{-0.084}^{+0.076}$ & $R_{*}$ \\
\hline Transit duration $W$ & $0.0589_{-0.0023}^{+0.0026}$ & $0.0593_{-0.0023}^{+0.0029}$ & $0.0612 \pm 0.0039$ & $0.0607_{-0.028}^{+0.025}$ & days \\
\hline Orbital inclination $i$ & $81.7_{-1.0}^{+1.2}$ & $81.8_{-1.0}^{+1.4}$ & $82.8_{-1.8}^{+2.6}$ & $82.5_{-1.3}^{+1.4}$ & deg \\
\hline Planet radius $R_{\mathrm{p}}$ & $2.21_{-0.16}^{+0.15}$ & $2.20 \pm 0.12$ & $2.04_{-0.14}^{+0.15}$ & $2.173_{-0.098}^{+0.097}$ & $R_{\oplus}$ \\
\hline
\end{tabular}
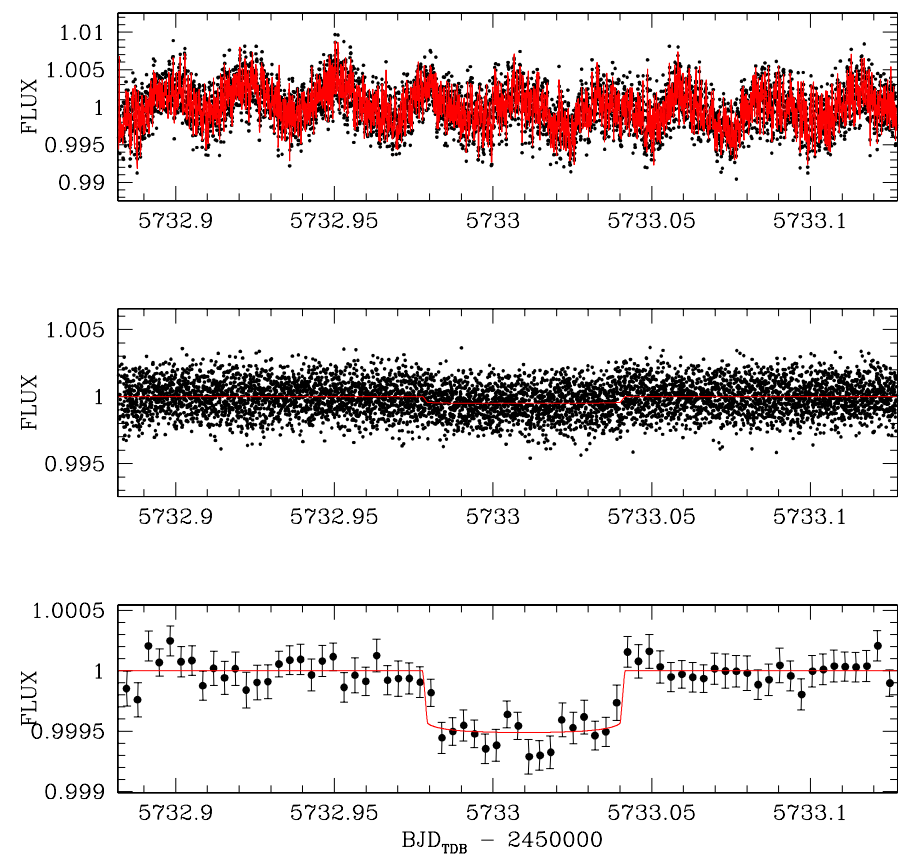

Fig. 2. Top: raw light curve for the second transit of 55 Cnc e observed by Warm Spitzer, with the best-fit global model superimposed. Middle: same light curve after division by the best-fit baseline model, with the best-fit transit model superimposed. Bottom: idem after binning per intervals of $5 \mathrm{~min}$.

The best-fit transit model and the detrended light curves are visible in Fig. 3. Table 2 presents the resulting values and errors for the transit parameters. We notice from this table that including the first transit as input data improves the global precision on the planet's size, but not on the transit duration/impact parameter, because of the degeneracy between the transit duration and the increase of the effective gain that affected the first timeseries.

\subsection{Independent analysis of the MOST data}

To assess the consistency of our Spitzer data with the MOST photometry, we performed our own analysis of the MOST time-series based on a different strategy from that used by W11. These authors analyzed the MOST data in two steps. First, they corrected their photometry from several instrumental effects known to affect MOST photometry. In a second step, they folded the corrected light curve on the orbital period of $55 \mathrm{Cnc}$, binned it to time intervals of $2 \mathrm{~min}$, and performed a MCMC analysis of the resulting light curve. To be consistent with our analysis of the Spitzer data, we decided on our side to use the raw MOST light curve (Winn, priv. comm.) as input data and to include a model for the systematic effects affecting MOST photometry in our global modeling. This strategy has the advantage to ensure the proper error propagation from the baseline model parameters to the transit parameters while avoiding any bias of a preliminary detrending/pre-whitening process on the derived results.

We thus started from the 27950 photometric measurements gathered by MOST between 07 Feb. 2011 00h15 to 22 Feb. 2011 00h05 UT. For each photometric measurement, we also had the corresponding background counts, PSF center position on the MOST CCD, a calculated magnetic field, and several other external parameters. Following W11, we rejected measurements obtained with a different exposure time from the bulk of the data, $41.82 \mathrm{~s}$. We also rejected measurements with an $x$ - or $y$-position off by more than 0.5 pixel from the median of the corresponding distributions. We indeed noticed a strong dependance of the measured fluxes to the PSF center positions, so we chose to discard discrepant measurements in terms of position to improve our chances to model the position effect with a simple analytical function. We also rejected outlier measurements with a magnitude off by more than 0.01 mag compared to the median value. We then tested several models to represent the data satisfactorily, our goal being at this stage not to find an optimum model but to identify discrepant measurements caused by transient effects (e.g. cosmic rays). Once we had selected such a model and fitted it to the data, we analyzed the residuals and performed a 10-sigma clipping to reject outliers. The resulting light curve was then binned with intervals of $5 \mathrm{~min}$ for the sake of computational speed, resulting in 3232 flux measurements.

We then began to iterate on models to represent the data in the best way. We noticed that the correlation of the fluxes and the positions could be satisfactorily modeled by an $x$ - and $y$-position polynomial. We also noticed a strong dependence of the measured fluxes with the background counts and the magnetic field which we were also able to model with polynomial functions of these external parameters. Once detrended from the position, magnetic field and background effects, the data revealed after a Lomb-Scargle periodogram analysis (Press et al. 1992) significant power peaks at $\sim 1.7 \mathrm{~h}, \sim 0.94$ days and $\sim 0.72$ days, with false-alarm probabilities $<10^{-11}$ for the three periods. The first period corresponds to the orbital period of the MOST satellite (W11), while the second and third are close to the rotation period of the Earth and, interestingly, to the orbital period of $55 \mathrm{Cnc}$, respectively. After filtering the residuals from these periodic signals by fitting sinusoids at the corresponding periods, the resulting light curve showed power excess at lower frequencies. We averaged them by dividing the light curve into five light curves 

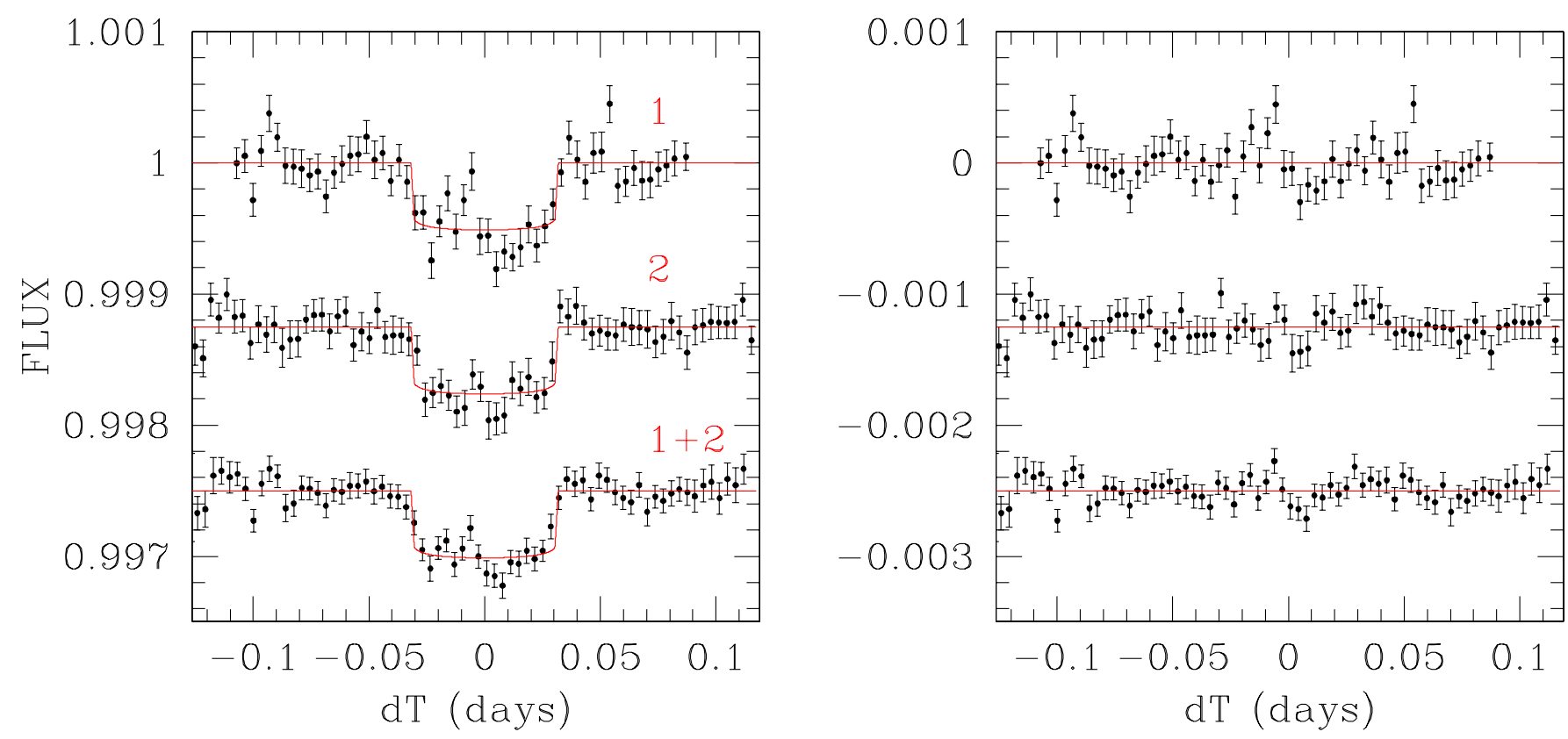

Fig. 3. Left: Warm Spitzer 55 Cnc light curves (1 and 2: individual transits, $1+2$ : combined light curve) divided by their best-fit baseline models deduced from their global MCMC analysis, binned to intervals of $5 \mathrm{~min}$, with the best-fit transit model superimposed. Right: residuals of the fit binned to intervals of $5 \mathrm{~min}$. For both panels, two time-series were shifted along the $y$-axis for the sake of clarity.

covering nearly equal durations, which we analyzed globally, assuming a different baseline for each of them.

We then performed a thorough model comparison as described above for Spitzer data to select the best model to represent the five resulting light curves. The baseline model that we selected contained a third-order $x$ - and $y$-position polynomial, a fourth-order background polynomial, a fourth-order magnetic field polynomial, a fourth-order time polynomial, one sinusoid at the satellite orbital period and one at a period of $\sim 0.94$ days. The transits of $55 \mathrm{Cnc}$ e were modeled with the formalism of Mandel \& Agol (2002) assuming a quadratic limb-darkening law and using the Gaussian priors on the coefficients $u_{1}$ and $u_{2}$ shown in Table 1. In addition, we also introduced a model for the flux modulation at the orbital period of $55 \mathrm{Cnc}$. We represented this "phase curve" with a simple model dividing the planet into four equal slices and assuming for each of them a uniform luminosity and a 1:1 spin-orbit resonance for the planet. For each slice, the flux modulation was modeled by a simple sinusoid with its maximum corresponding to the center of the slice pointing toward Earth. We emphasize that our goal here was to select a model that satisfactorily represents the flux modulation without worrying about its physical relevance. Indeed, the amplitude of the observed flux modulation is much too large to be attributed to the variable illuminated fraction of the planet or to its thermal emission, as outlined by W11. We tested introducing an occultation in our global model, but it did not improve the model marginal likelihood, consequently we discarded it from our final modeling.

In our complex model, the best-fit residuals of the five light curves still show a small amount of correlated noise that we took into account in the same way as for Spitzer data by rescaling the measurements errors. At this stage, we performed a global analysis of our five light curves and performed for this purpose two MCMC chains of 100000 steps, whose convergence we successfully checked for the transit parameters using the statistical test of Gelman \& Rubin (1999). Table 2 provides the resulting values and 1- $\sigma$ error bars for the transit and physical parameters, while Fig. 4 displays the best-fit models (global, phase curve + transits, transit) superimposed on the corresponding light curves.

We notice that our results are consistent with W11's results, the agreement being at better than $1-\sigma$ for the transit depths and $\sim 1.5-\sigma$ for the transit impact parameters. Considering the large difference between both analyses, notably the different treatment of systematic effects, this agreement is reassuring for the robustness of the resulting inferences. As described above, we divided the MOST data into five light curves, which were treated separately from the others in our global MCMC analysis, except for the transit parameters. The free parameters for the phase curve model, i.e. the amplitudes of the sinusoid of each planet slice, were thus different for the five light curves. Interestingly, Fig. 4 shows that the five best-fit phase curve models show some variability (see discussion in Sect. 4.2).

\subsection{Global analysis MOST + Spitzer data}

As can be noticed in Table 2, our independent analysis of Spitzer and MOST data led to consistent results and similar precisions on the planet radius. For both instruments, the limiting factor on the transit depth precision is not the white noise associated with the flux measurements but the high level of systematic effects that affect the photometry. Aiming to minimize the impact of these systematics and to improve the precision on the transit's shape and the planet's size even more, we performed a global analysis of MOST and Spitzer data, assuming that the transit depth is exactly the same in both channels, i.e. that chromatic atmospheric transmission effects are not significant at this level of photometric precision. This is an entirely reasonable assumption, considering the expected small atmospheric scale height of the planet (see Sect. 4.2 for more details). The used priors, baseline models and analysis details were the same as in the separate analysis described above. Table 2 presents the resulting values and 1- $\sigma$ error bars for the transit and physical parameters.

Finally, we assessed the validity of the assumption that both channels probe the same transit depth by performing a new 
M. Gillon et al.: Improved precision on the radius of the nearby super-Earth $55 \mathrm{Cnc}$ e
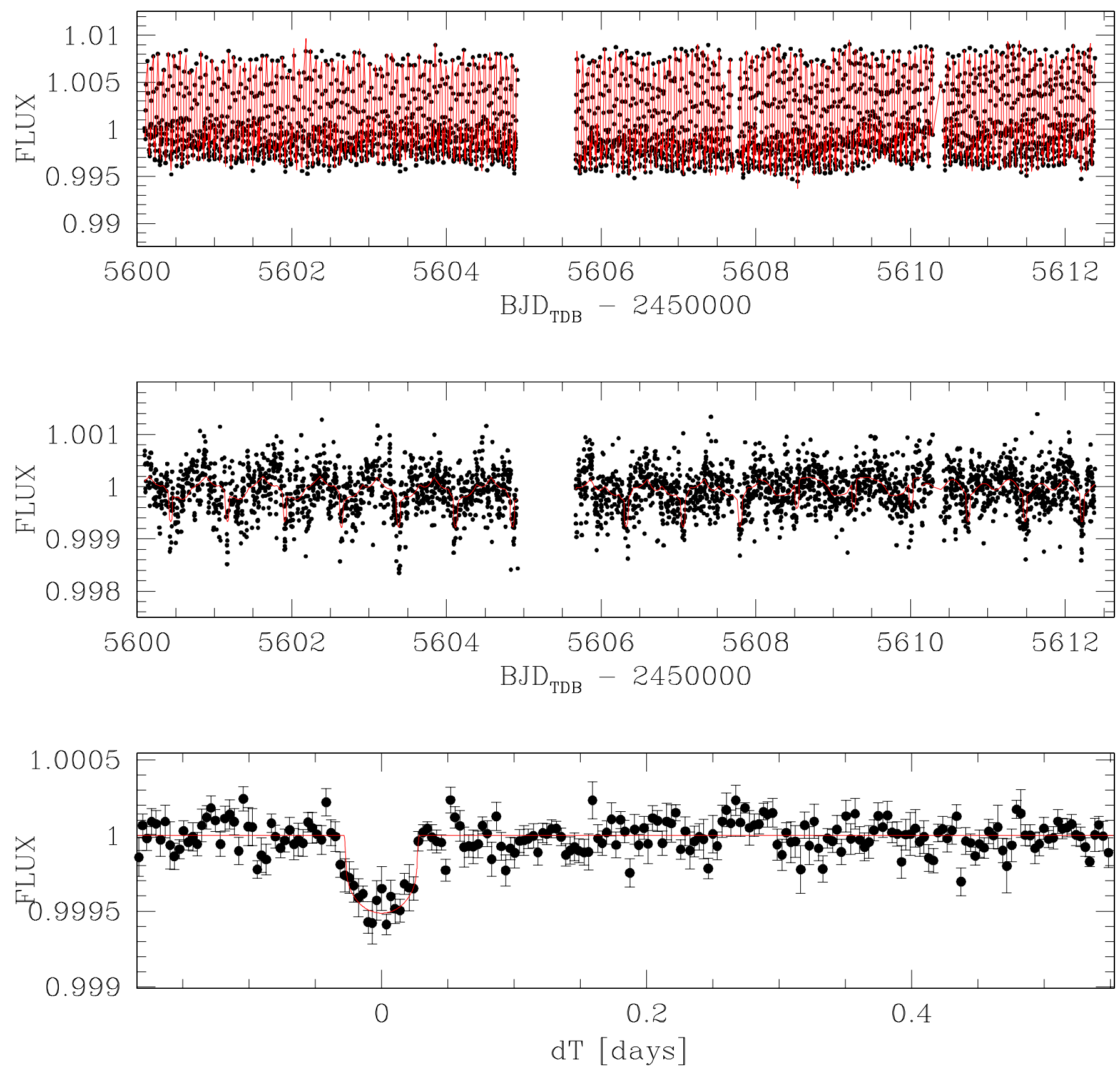

Fig. 4. Top: 55 Cnc MOST raw photometry with our best-fit global model superimposed. Middle: MOST photometry divided by the best-fit baseline model, and with the best-fit transit + phase-curve model superimposed. Bottom: MOST photometry divided by the best-fit baseline + phase curve model, folded with the best-fit orbital period of $55 \mathrm{Cnc}$ e and binned per $5 \mathrm{~min}$ intervals, with the best-fit transit model superimposed.

global analysis of MOST and Spitzer data, this time adding as free parameter a difference in transit depth between the two instruments. The derived transit depth difference Spitzer - MOST was $94 \pm 80 \mathrm{ppm}$, i.e. the infrared radius of the planet is consistent with the optical radius, as already deduced from the individual analysis of the data of both instruments. The other deduced transit parameters were very similar to those shown in Table 2.

\section{Discussion}

\subsection{The composition of 55 Cnce}

To infer the composition of the planet we used the internal structure model by Valencia et al. $(2006,2010)$ and considered representative compositions for rocky and volatile planets. The range in radii for rocky planets are delimited by a pure iron composition that yields the smallest radius and a magnesium-silicate oxide composition (devoid of iron) that yields the largerst radius. Owing to the fact that iron, magnesium, and silicate are all refractory elements with similar condensation temperatures, planets are unlikely to form with either of these extreme compositions. Two plausible compositions we looked at are Earth-like composition (33\% iron core above a silicate mantle with $10 \%$ of iron and $90 \%$ of silicate by mol) and an iron-enriched composition (63\% iron core above a Mg-Si mantle, with no iron).

We find that $55 \mathrm{Cnc}$ e is too large to be made out of just rocks despite its relative high bulk density of $\rho=4.0_{-0.3}^{+0.5} \mathrm{~g} \mathrm{~cm}^{-3}$ (e.g. Earth's bulk density is $\rho_{\oplus}=5.5 \mathrm{~g} \mathrm{~cm}^{-3}$ ) obtained with the radius 


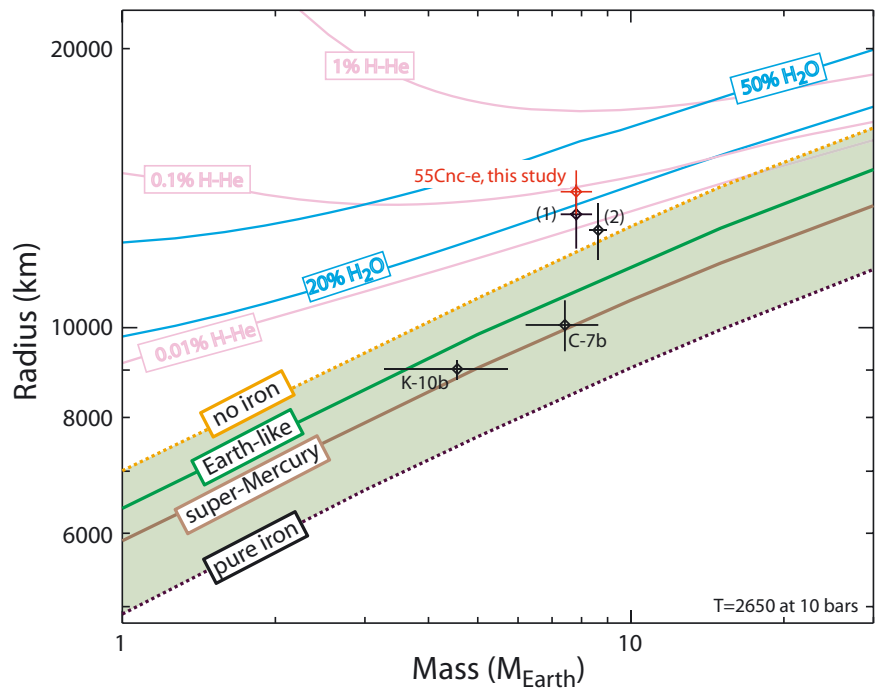

Fig. 5. Composition of $55 \mathrm{Cnc}$ e. Mass-radius relationships for four different rocky compositions: pure iron, super-Mercury (63\% iron core above a $37 \%$ silicate mantle), Earth-like (33\% iron core above a $67 \%$ silicate mantle with $10 \%$ iron by mol) and a silicate planet with no iron. The green band depicts the range of rocky compositions of planets between 1 and $30 M_{\oplus}$. Two families of volatile planets are considered: envelopes of $\mathrm{H}-\mathrm{He}$ with $0.01,0.1$ and $1 \%$ by mass (pink) and envelopes of water with $20,50 \%$ water. Kepler- $10 \mathrm{~b}(\mathrm{~K}-10 \mathrm{~b})$ and CoRoT-7b (C-7b) are shown for reference. Data for $55 \mathrm{Cnc}$ e from this study are shown in red, and are taken from D11 (1) and W11 (2).

reported in this study. Therefore, it has to have an envelope of volatiles. We consider two compositions for the gaseous envelope: a hydrogen and helium mixture, and a pure water vapor composition (which at these temperatures is super-critical). We added different amounts of envelope to an Earth-like nucleus. The results show (see Fig. 5) that the data may be fitted with a $\mathrm{H}-\mathrm{He}$ envelope of $\sim 0.1 \%$ by mass or a $\mathrm{H}_{2} \mathrm{O}$ envelope of $\sim 20 \%$ by mass. As described in D11, based on simple atmospheric escape calculations described in Valencia et al. (2010), this low-mass envelope of H-He would escape in Myr timescales, whereas a water-vapor envelope would escape in billions of years timescales. Thus, the favored composition for $55 \mathrm{Cnc}$ e is a volatile planet with a water dominated envelope comprising tens of percent of the total mass of the planet.

The radius obtained in this study is larger than that reported by D11 and above a one-sigma level of W11 value, ruling out a rocky composition for $55 \mathrm{Cnc}$. An interesting characteristic of this planet is that it has an intermediate composition (see Fig. 6). While most of its mass is bound in a rocky nucleus, it has a non-negligible (most likely high-molecular) envelope. This lies between the composition of GJ $1214 \mathrm{~b}$, which probably consists mostly of water (see Valencia et al. 2011), and the terrestrial planets in our solar system and exoplanets such as "super-Mercuries" CoRoT-7 b (Hatzes et al. 2011) and Kepler10 b (Batalha et al. 2011).

\subsection{Potential atmospheric studies of 55 Cnce}

Follow-up observations made to detect the spectral signature of the planetary atmosphere may bring new constraints on the chemical composition of $55 \mathrm{Cnce}$. The planet is suitable for these observations, because it orbits around a very bright, nearby star at an extremely close distance. Still, depending on the nature

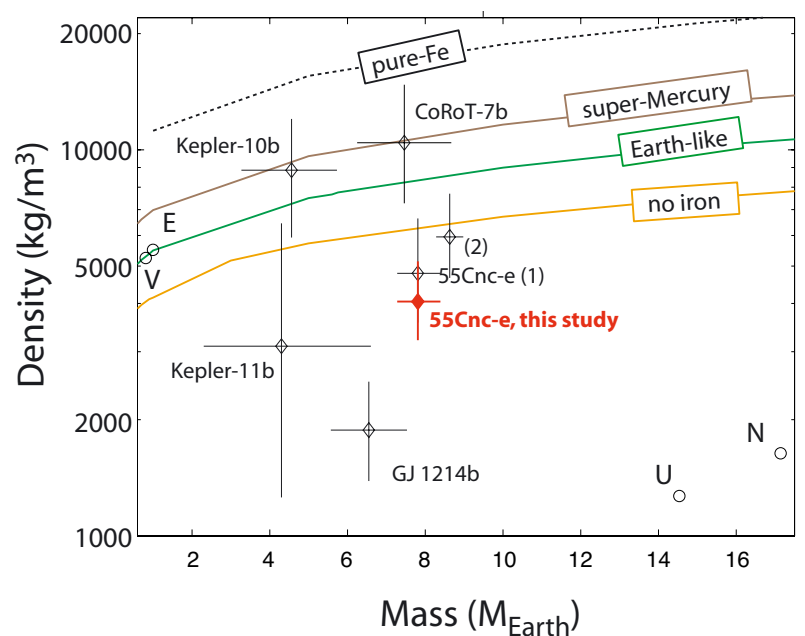

Fig. 6. Density-mass relationships of low-mass exoplanets. Four rocky compositions are shown (see Fig. 5 for description). 55 Cnc e's radius from this study shows the planet cannot be rocky and instead is intermediate between the envelope-rich planets (e.g. GJ 1214 b) and the rocky ones. Earth, Venus, Uranus, Neptune, Kepler-11 b, GJ 1214 b, Kepler$10 \mathrm{~b}$, and CoRoT-7 b are shown for reference.
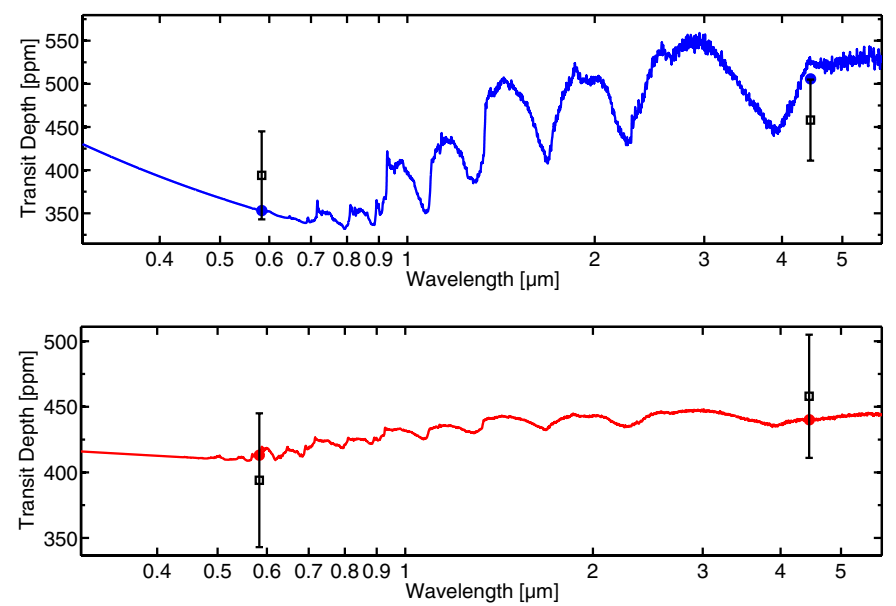

Fig. 7. Broadband transit depth measurements from MOST and Spitzer (squares) compared to models (lines + filled circles). Theoretical transmission spectra for $55 \mathrm{Cnc}$ e are displayed for an atmosphere composed of $100 \%$ water vapor (bottom) and an atmosphere with solar composition (top). The model assumes a gravitationally bound atmosphere in radiative-convective equilibrium (Benneke \& Seager 2011).

of the planetary atmosphere, follow-up observations can be extremely challenging due to the shallow transit depth.

Atmospheric modeling (Fig. 7) suggests that transmission signatures of an atmosphere in hydrostatic equilibrium could be on the order of $100 \mathrm{ppm}$, if the planet has been able to accrete and retain a hydrogen-dominated atmosphere. Such signatures could be detected or excluded with currently available space-based instrumentation. However, the short evaporation timescale for a $\mathrm{H}-\mathrm{He}$ envelope strongly disfavors this scenario, as stated above. The most probable scenario implies an envelope mostly composed of water and other ices which would result in much weaker transmission signatures on the level of tens of ppm due to the higher mean molecular mass (Fig. 7). A direct detection of such a water/ices dominated atmosphere on 55 Cnce will probably have to wait until next generation instruments on-board JWST become available. 
Stronger transmission signatures are plausible, if one hypothesizes that the planet is surrounded by a low-density halo of atomic gas resulting from atmospheric escape. Both the small Roche lobe and the high equilibrium temperature favor a strong atmospheric escape and the lost atmospheric mass could be readily replenished by evaporation of the planet's surface or oceans (Yelle et al. 2008; Schaefer \& Fegley 2009; Ehrenreich 2010). The evaporated gas should be readily dissociated in the hightemperature halo. The resulting atomic species like $\mathrm{C}, \mathrm{H}, \mathrm{O}, \mathrm{Na}$, $\mathrm{Mg}$ and $\mathrm{Ca}$ could be detectable in transmission because of their strong absorption cross sections near their electronic transition lines and the large extent of the halo (e.g. Mura et al. 2011). The exosphere of 55 Cnc e could also explain the flux modulation at the planet's orbital period detected by W11 and our own analysis of MOST data. Because the amplitude of this modulation is too large for the planet's thermal emission or reflected light, W11 hypothesized the induction by the planet of a patch of enhanced magnetic activity on the star. Another explanation is that a part of the gases escaped from the planet's atmosphere forms a circumstellar disk along the planet's orbit similar to Io's donut-shaped cloud (e.g. Schneider \& Bagenal 2007). The short lifetime of these evaporated gases would modulate the opacity of this cloud along the orbit. Furthermore, the produced ionic species would rotate with the star magnetic field, leading to a modulation of the resulting "phase curve" at the rotational period of the star, $42.7 \pm 2.5$ days (Fischer et al. 2008), which is consistent with the apparent variability of the fitted phase curve model in our analysis (Fig. 4, middle panel). More work is needed to assess the plausibility of this hypothesis.

Because of its extreme proximity to its host star and the resulting incident flux of $\sim 3.3 \times 10^{9} \mathrm{erg} \mathrm{s}^{-1} \mathrm{~cm}^{-2}$, 55 Cnce's thermal emission could be measured through infrared occultation photometry. With realistic assumptions for the Bond albedo and heat distribution efficiency of the atmosphere, occultation depths ranging between 90 and $150 \mathrm{ppm}$ are expected at $4.5 \mu \mathrm{m}$. The photometric precision demonstrated here shows that a lowamplitude eclipse like this could be detected by Spitzer, provided several events are monitored. This is the goal of our accepted Spitzer program 80231, and we are waiting eagerly for these future observations to learn more about this fascinating planet.

Acknowledgements. We are grateful to J. Winn for having provided us with the MOST photometry. This work is based in part on observations made with the Spitzer Space Telescope, which is operated by the Jet Propulsion Laboratory, California Institute of Technology under a contract with NASA. Support for this work was provided by NASA. We thank the Spitzer Science Center staff for efficient scheduling of our observations. M. Gillon is FNRS Research Associate.

\section{References}

Batalha, N. M., Borucki, W. J., Bryson, S. T., et al. 2011, ApJ, 729, 27 Benneke, B., \& Seager, S. 2011, ApJ, submitted

Carlin, B. P., \& Louis, T. A. 2008, Bayesian Methods for Data Analysis, third edition (Chapman \& Hall/CRC)

Chib, S., \& Jeliazkov, I. 2011, JASA, 96, 453

Claret, A., \& Bloemen, S. 2011, A\&A, 529, A75

Demory, B.-O., Gillon, M., Deming, D., et al. 2011, A\&A, 533, A114 (D11)

Ehrenreich, D. 2010, EAS Pub. Ser., 41, 12

Fischer Fischer, D. A., Marcy, G. W., Butler, R. P., et al. 2008, ApJ, 675, 790

Gelman, A., \& Rubin, D. 1992, Stat. Sci., 7, 457

Hatzes, A. P., Fridlund, M., Nachmani, G., et al. 2011, ApJ, 743, 75

Knutson, H. A., Charbonneau, D., Allen, L. A., et al. 2008, ApJ, 673, 526

Mandel, J., \& Agol. E. 2002, ApJ, 580, 171

Mura, A., Wurz, P., Schneider, J., et al. 2011, Icarus, 211, 1

Press, W. H., Flannery, B. P., Teukolsky, S. A., \& Vetterling, W. T. 1992, Numerical Recipes in Fortran, 2nd edn. (Cambridge University Press) Schaefer, L., \& Fegley, B. 2009, ApJ, 703, L113

Schneider, N. M., \& Bagenal, F. 2007, Io After Galileo: A New View of Jupiter's Volcanic Moon (Germany: Springer), 265

Seager, S., Deming, D., \& Valenti, J. A. 2009, Transiting Exoplanets with JWST, Astrophysics in the Next Decade, Astrophysics and Space Science Proc. (Netherlands: Springer), 123

Valencia, D. 2011, in The Astrophysics of Planetary Systems: Formation, Structure and Dynamical Evolution, IAU Proc., 276, 181

Valencia, D., O'Connell, R. J., \& Sasselov, D. 2006, Icarus, 181, 545

Valencia, D., Ikoma, M., Guillot, T., \& Nettelmann, N. 2010, A\&A, 516, A20 Valenti, J. A., \& Fischer, D. A. 2005, ApJS, 159, 141

von Braun, K., Boyajian, T. S., ten Brummelaar, T. A., et al. 2011, ApJ, 740, 49

Winn, J. N., Matthews, J. M., Dawson, R. I., et al. 2011, ApJ, 737, L18 (W11)

Yelle, R., Lammer, H., \& Wing-Huen, Ip 2008, SSRv, 139, 437 